\title{
AN IMPROVED PERIMETER AND SCREEN FOR EXAMINING THE FIELD OF VISION BY
}

DR. H. LEWKOWITSCH, LONDON.

THE improvements I propose to introduce in the construction and the use of the perimeter or the Bjerrum screen, seem to be of such nature as to diminish greatly the apparent limitations

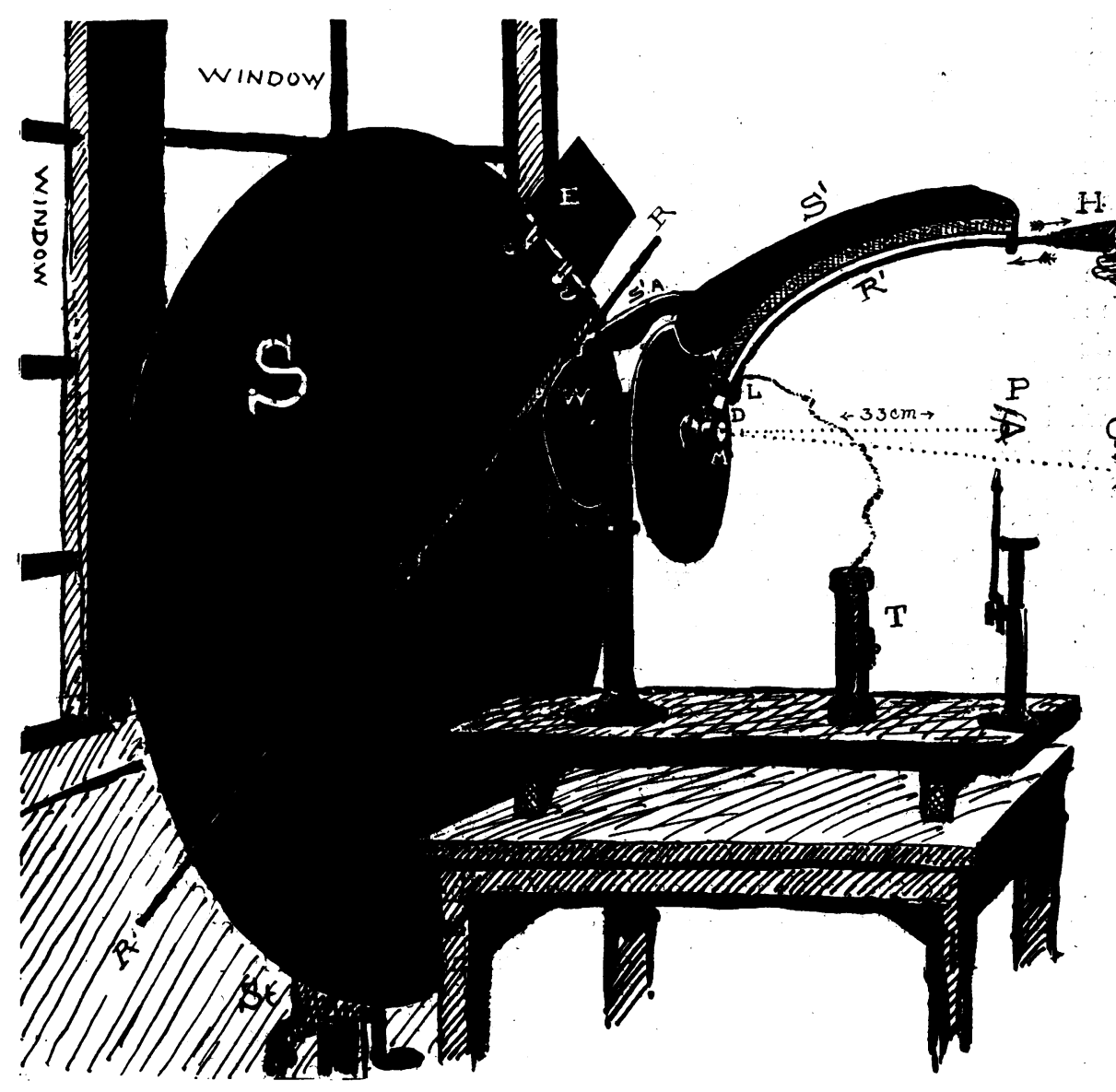

which were so far unavoidably attached to the use of the two above-mentioned instruments in their present state of construction. The three drawings given in Figs. 1, 2, 3 speak for themselves, and require only few additional explanations. Fig. 1 illustrates a perimetric examination with the aid of a Priestley Smith perimeter. To this latter instrument I added an additional broader background, 
represented by the meridionally-curved screen $\mathrm{S}^{\prime}$, a black flap. $\mathrm{S}^{\prime}$ is fixed by a bent blackened side-arm (S'A) to the disc W, while the other end of $S^{\prime}$ is tied to the foremost end of the graduated perimeter arc. W, S'A, and $S^{\prime}$ rotate conjointly with the perimeter arc when measuring the spherical field of vision. In the centre of the perimeter is placed the mirror $(\mathrm{M})$, which, to my mind, constitutes the most essential and pre-eminent part of the improvements here introduced. For this reason I may be allowed to

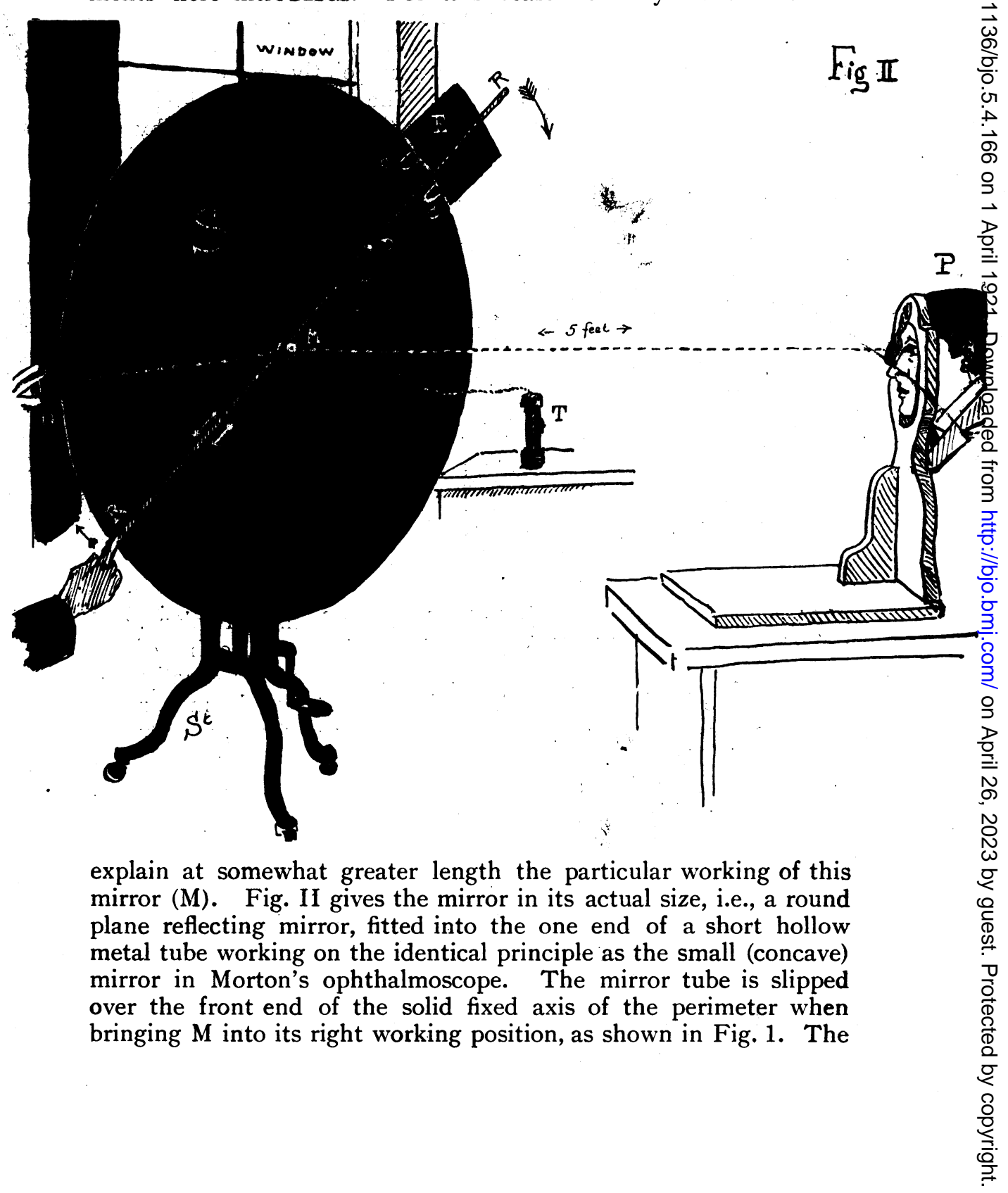




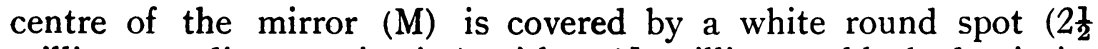
millimetres diameter in size) with a $1 \frac{1}{2}$ millimetre black dot in its middle, these two marks constituting the fixed object to be fixed by the patient. The movable mark D (Fig. 1) a white disc, is fixed together with the illuminating minute electric lamp L-(L being enclosed in a small blackened metal encasement) - to the inner end of the rod ( $\left.R^{\prime}\right)$. The outer end of the rod ( $\left.R^{\prime}\right)$ passes through the opening of a metal ring-(this ring being fixed to the outer end of the perimeter arc) - terminating in the handle $(\mathrm{H})$. By pushing the handle $(\mathrm{H})$ inwards and pulling it outwards, the observer makes the rod ( $\left.R^{\prime}\right)$ glide along the graduated arc conjointly and consensually with the disc (D) and the lamp (L). The movement of the handle $(\mathrm{H})$ or of the hand of the observer remain unobserved by the patient, while the gliding movement of $R^{\prime}$ along the arc can be carried on so as to remain almost entirely inaudible. P., the eye of the patient, who is resting with his chin on the chin rest, is fixing the mirror (M) from a distance of $33 \mathrm{~cm}$., while $O$ is the position of the eye of the observer, who is sitting behind, and slightly sideways of, the patient, looking over the patient's shoulder towards the centre

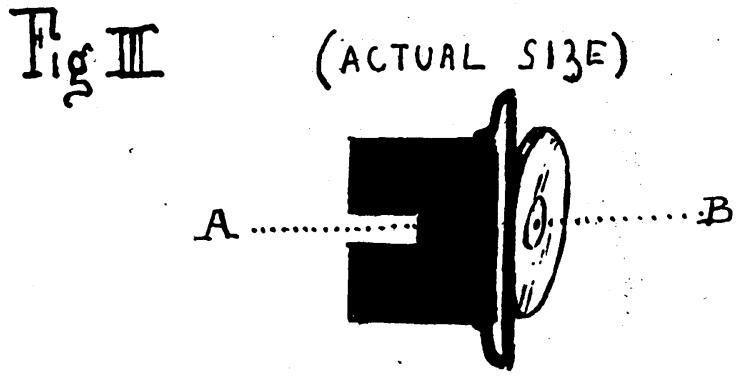

of M. When PMO are exactly in the same horizontal plane, and the observer keeps his line of fixation strictly to the line of the reflected ray (true to the angle $\mathrm{PMO}$ ), he can see exactly the patient's eye behind the black dot in the centre of the mirror (M) and he can control all and any, even the very slightest, movements of the patient's pupil. Thus, to my mind, all the measurements in perimetric observations are made to a very high degree true, definite, and reliable; while the ordinary method of direct observation, fixing more or less accurately the patient's eye, is bound to lead to greater or lesser errors of calculation. My method naturally finds its limitation where the patient's vision is so defective that he cannot fix at all the centre of the mirror. Accordingly, as the observer examines the right or the left hemisphere of the field of vision, he should take his position alternately behind the patient's right or left shoulder, and the observer should at the same time turn the (oblique standing) mirror $\mathrm{M}$ (cf. Fig. 3.) round the axis $\mathrm{AB}$ by an 
angle of $180^{\circ}$. Fig. 1 shows how the perimeter observation is made by the additional aid of a large Bjerrum screen (S) placed at the same time behind the perimeter and in front of two windows, hereby allowing only light coming chiefly sideways from right and left on the patient's eye. $\mathrm{P}$ is thus strongly illuminated and shining up brilliantly in the mirror against the large black ground of S.

Fig. II illustrates the observation made with the screen (S) alone. The radius of $\mathrm{S}$ is only 2 feet (so as to make the screen less bulky), and there is an additional ( $\frac{3}{4} \mathrm{ft}$. size) screen extension (E), easily to be attached by two black clips (CC) to the outer ring circumference of the screen (S), protruding along the line of the meridian, along which the measurement just happens to be carried out by the rod. The screen (S) is fixed to the top of an adjustable ophthalmic table (ST), so that $\mathrm{S}$ remains in a fixed position, in which it cannot be turned. In front of the plane of (S) protrudes slightly the streen-axis (as shown in Fig. 1) in the shape of a hollow metal tube perforated transversely by two small round openings, which just allow the black (graduated) rod $(\mathbf{R})$ to pass through in a gliding radial movement and to rotate also round the screen-axis (as shown by arrows in Fig. II). A thick cork (of about $1 \frac{1}{4}$ inch length) is fitted to half its length into the front opening of the screen axis tube, when by pressing the cork tighter and deeper into the tube towards the passing rod, the latter can be arrested hereby in any position. Over the protruding end of the cork is then slipped the tube end of the mirror (M), which latter is then fixed in this position (cf. Fig. II) to be handled and used in the same way as described in the perimeter examination. The observer stands in this case in front of the screen, slightly to the right or left of the centre of $S$ with his back to the patient, watching all the time the patient's eye as reflected in the mirror (M) in the direction of OM. The observer is then shifting with his one hand radially and by a rotating movement the one end of the rod (R) and conjointly and consensually with it at the same time also the disc (D) and the lamp and lampholder (L) These three objects being jointly fixed to the rod (R). The other disengaged hand of the observer manipulates the switch of the electric torch (T), which is to be carried, for greater convenience, in the observer's breast pocket during the perimetric as well as the screen observation. If absolute darkness of the room be preferred for the observation, instead of the daylight illumination; one should post a small electric lamp to the right or left side at the back of the screen (S), allowing only a narrow pencil of light passing from the lamp in the direction of the patient's eye.

The improvements above suggested may be readily adapted to Elliot's scotometer. 